\title{
OCULAR MANIFESTATIONS OF CORONAVIRUS DISEASE (COVID 19) AT A TERTIARY CARE HOSPITAL IN EASTERN INDIA
}

\section{Ophthalmology}

Sushmita Mukherji Senior Resident, Regional Institute of Ophthalmology, Kolkata, West Bengal, India

\author{
Sibaji Dasgupta*
}

Consultant Neurosurgeon, National Neurosciences Centre, Kolkata, West Bengal, India*Corresponding Author

\section{Soumen Karmakar Consultant Psychiatrist, West Bengal, India}

\section{ABSTRACT}

Background: A Worldwide concern, Coronavirus disease 2019 (COVID-19) which is caused by a novel coronavirus termed severe acute respiratory syndrome coronavirus-2 (SARS-CoV-2), has been also found to be associated with ocular signs and symptoms. Apart from being transmitted among close contacts via respiratory droplets, it is being considered that ocular mucosa, its secretions can also be responsible for transmission. Aims and objectives: To see the different ocular manifestations in patients admitted with COVID-19 infection at a tertiary care hospital along with presence of the virus in ocular secretions. Materials and Methods: This was a prospective study on 80 patients admitted with SARS-CoV-2 infection diagnosed by Reverse transcriptase-polymerase chain reaction (RT-PCR) positive results and followed up during their hospital stay in COVID isolation wards, till discharge or death within a time period from 15th April, 2020 to $10^{\text {th }}$ August,2020. Results: Out of the 80 SARS-CoV-2 positive patients studied, 10(12.5\%) patients had conjunctival chemosis, 8(10\%) patients had conjunctival hyperaemia,12(15\%) patients had epiphora and $9(11.25 \%)$ patients had increased ocular discharge. $5(6.25 \%)$ patients had positive results for SARS-CoV-2 RT-PCR in both conjunctival as well as nasopharyngeal swabs. Conclusion: Ocular involvement can be one of the different systemic involvements of SARS$\mathrm{CoV}-2$ infection and ocular secretion can be a potential source of transmission of the virus. Hence, ocular protection in the form of spectacles or face shields must be undertaken apart from the other barriers to reduce the possible chances of transmission of the disease.

\section{KEYWORDS}

\section{COVID-19; SARS-CoV-2; RT-PCR; Ocular involvement.}

\section{INTRODUCTION}

A novel coronavirus, SARS-CoV-2, emerged in China in the city of Wuhan in December 2019. On March 11, 2020 the World Health Organization declared the COVID-19 outbreak a pandemic. The number of cases has been dramatically increasing because of the highly contagious nature of the disease and lack of definitive / effective treatments. It is yet too early to predict an actual time to the certain control and as a result an end to this pandemic. Another coronavirus, named SARS-CoV-1, was responsible for severe acute respiratory syndrome.2 Compared to SARS-CoV-1, SARS-CoV-2 has a similar binding receptor and similar pathologic features systemically and epidemiological characteristics.2,3 Although there is no direct evidence that SARS-CoV-1 replication results in conjunctivitis and other ocular diseases, reports have emphasized the eye as a potential site for virus transmission. ${ }^{4}$ The objective of this study was to evaluate similar ocular involvement in patients who were confirmed to have COVID-19 infection.

\section{METHODS}

In this prospective study, we followed up Consecutive samples of 80 patients who were diagnosed as COVID-19 positive by means of reverse transcriptase-polymerase chain reaction (RT-PCR) from nasopharyngeal swabs and admitted in Covid isolation wards at a tertiary care hospital in Kolkata. Prior approval from Institutional Ethics Committee and Proper consent from patients(all adults and able) were taken. The study started on 15th April,2020 and concluded on10th August,2020. During this period we followed up those patients upto their discharge or death. Ocular symptoms were noted and superficial torch light examinations were performed. RT-PCR from conjunctival swabs for SARS-CoV-2 were performed and analyzed. Detailed examination involving anterior segment details by slit lamp and subsequent fundus examination could not be done as those facilities were not available in the COVID wards. Patients were graded according to the guideline of PC-NCP5: the category-Mild, indicated clinical symptoms were mild and there was no sign of pneumonia on imaging. Moderate indicated fever and/or respiratory symptoms and lung computed tomography imaging findings; Severe indicated dyspnoea (respiratory frequency of 30 cycles per minute or greater), blood oxygen saturation of $93 \%$ or less, and an arterial partial pressure of oxygen to fraction of oxygen inspiration ratio of 300 or less; and Critical indicated respiratory failure or shock or multiple organ dysfunction/failure.

\section{RESULTS}

Out of the 80 COVID-19 positive patients, 30(37.5\%) patients developed ocular signs and symptoms. Epiphora was found in $12(40 \%)$ patients, Conjunctival chemosis was found in 10(33.33\%) patients, increased discharge was found in $9(30 \%)$ patients, conjunctival hyperaemia was found in $8(26.66 \%)$ patients. $5(6.25 \%)$ patients had positive results of SARS-CoV-2 in conjunctival swabs along with nasopharyngeal swabs. 60 patients had mild pneumonia of which $20(33.33 \%)$ had ocular involvement, 12 patients had moderate pneumonia of which $3(25 \%)$ had ocular involvement, 5 patients had severe pneumonia of which $4(80 \%)$ had ocular involvement and all the 3 patients with critical pneumonia had ocular involvement (100\%).

TABLE:1 Frequency of different ocular involvements in COVID positive patients

\begin{tabular}{|c|c|}
\hline Ocular involvement & No. of cases (\%) \\
\hline Epiphora & $12(40 \%)$ \\
\hline Conjunctival chemosis & $10(33.33 \%)$ \\
\hline increased discharge & $9(30 \%)$ \\
\hline conjunctival hyperaemia & $8(26.66 \%)$ \\
\hline
\end{tabular}

COMPOUND CHART: Frequency of ocular involvement in different Categories of patients

\section{Chart Title}

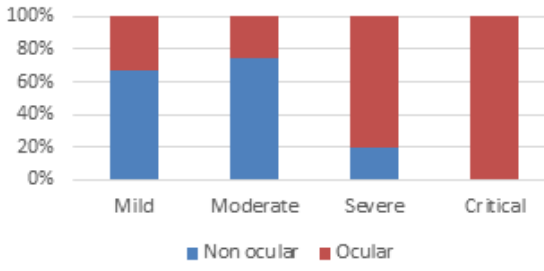

\section{DISCUSSION}

In this study we have tried to evaluate the pathological ocular manifestations of SARS-CoV-2 infection along with their relation with the severity of the disease. The first reported case of SARS-CoV-2 conjunctivitis was a member of the Chinese panel for pneumonia, who developed conjunctivitis and COVID-19 after performing an inspection in the Wuhan Fever Clinic without wearing eye protection. This case highlights the potential conjunctival transmission route. 6 $\mathrm{Wu}$ et al. performed a preliminary investigation about the prevalence of ocular manifestations in patients with COVID-19 and reported that chemosis, epiphora, and conjunctival hyperemia were present in onethird of the patients. Moreover, they observed that these manifestations 
most commonly occurred in patients with severe systemic disease. Overall, this study also suggested that even if only two conjunctival specimens $(5.2 \%)$ from patients with ocular abnormalities yielded positive findings for SARS-CoV-2 on reverse transcription polymerase chain reaction (RT-PCR), COVID-19 can potentially also be transmitted through the eyes.7 Our study also shows similar range of ocular manifestations along with presence of SARS-CoV-2 antigen in ocular secretions of some patients.

It has been be noted that those patients who need respiratory support in an ICU have high propensity to develop ocular complications. The incidence of eye-related complications in ICU patients in different studies varies from $3 \%$ to $60 \% .8$ Risk factors for developing conjunctival chemosis in critically ill include reduced venous return from the eye (due to positive pressure ventilation or tight endotracheal tube taping) and increased hydrostatic pressure (mainly due to prolonged recumbency, especially if prone). Since it increases venous pressure in the head, it can theoretically also cause subconjunctival hemorrhage, usually completely benign although it may lead to surface disorders, if extensive.9 In mechanically ventilated patients, the positive end-expiratory pressure may lead to subconjunctival hemorrhage because of an increase in intrathoracic pressure and consequently in central venous pressure.10 These possible mechanisms support our finding of having increased percentage of ocular complications in patients with severe and critical COVID-19 illness.

This study has got certain limitations. Complete follow up of patients were not done after discharge. Comprehensive ophthalmological examination of patients were also not possible in this study. Day of onset of symptoms were not analysed extensively. Larger sample size would have helped more to arrive in a proper conclusion.

\section{CONCLUSION}

Although adequate researches are yet to be published, the available studies till date including ours suggest an array of ocular complications in COVID-19 positive patients. The more severe the illness, the more chances of ocular complications. To add with, presence of the infectious agent in ocular secretions make it a greater concern. Hence adequate ocular protections in the form of spectacle goggles and face shields are required, apart from the facemasks and regular sanitization protocols to prevent transmission of this dreaded virus in the community as well as amongst healthcare providers.

\section{Acknowledgement}

None.

\section{Source of support}

None.

\section{Conflicts of interest}

The author declares there is no conflict of interest.

\section{REFERENCES}

1. Coronavirus covid-19 (2019-ncov). Availableat: https://gisanddata.maps.arcgis.com/ apps/opsdashboard/index.html\#/bda7594740fd40299423467b48e9ecf6 Google apps/op

2. Lu R, Zhao X, Li J, et al. Genomic characterisation and epidemiology of 2019 novel coronavirus: implications for virus origins and receptor binding. Lancet. 2020;395(10224):565-574. doi:10.1016/S0140-6736(20)30251-8PubMed

3. To KF, Lo AW. Exploring the pathogenesis of severe acute respiratory syndrome (SARS): the tissue distribution of the coronavirus (SARS-CoV) and its putative receptor, angiotensin-converting enzyme 2 (ACE2). J Pathol. 2004;203(3):740-743. doi:10.1002/path.1597PubMed

4. Peiris JS, Yuen KY, Osterhaus AD, Stöhr K. The severe acute respiratory syndrome. N Engl J Med. 2003;349(25):2431-2441. doi:10.1056/NEJMra032498PubMed

5. National Health Commission of the People's Republic of China. The guideline on diagnosis and treatment of the novel coronavirus pneumonia (NCP): revised version of diagnosis and treatment of the novel coronavirus pneumonia (NCP): revised version of
the 5th edition. Accessed February 8, 2020. http://www.nhc.gov.cn/xcs/zhengcwj/ 202002/d4b895337e19445f8d728fcafle3e13a.shtml

6. C. W. Lu, X. F. Liu, and Z. F. Jia, "2019-nCoV transmission through the ocular surface must not be ignored," The Lancet, vol. 395, no. 10224, p. e39, 2020.

7. Wu P, Duan F, Luo C, Liu Q, Qu X, Liang L, Wu K: Characteristics of ocular findings of patients with coronavirus disease 2019 (covid-19) in Hubei province, china. JAMA Ophthalmol, 2020. PMID: 32232433. DOI: 10.1001/jamaophthalmol.2020.1291

8. T. B. Saritas, B. Bozkurt, B. Simsek et al., "Ocular surface disorders in intensive care unit patients," Scientific World Journal, vol. 2013, Article ID 182038, 2013.

9. L. A. Lee, "Risk factors associated with ischemic optic neuropathy after spinal fusion surgery,"Anesthesiology, vol. 116, pp. 15-24,2012.

10. A. Abbas and G. Hyman, "Macular hemorrhage secondary to increased intrathoracic pressure and difficult intubation," JPMA. The Journal of the Pakistan Medical Association, vol. 52, no. 6, pp. 265-266,2002 